\title{
Synthesis of substituted 2,5-dihydro-1-naphthoxepines from 1- naphthol via ring-closing metathesis
}

\author{
Jui-Chi Tsai, ${ }^{a}$ Sie-Rong Li, ${ }^{a}$ Liang-Yeu Chen, ${ }^{a}$ Po-Yuan Chen, ${ }^{b}$ Sodio Chih-Neng Hsu, \\ Chun-Nan Lin, ${ }^{a}$ and Eng-Chi Wang ${ }^{b_{*}}$ \\ ${ }^{a}$ Institute of Pharmaceutical Sciences, Kaohsiung Medical University, Kaohsiung City, Taiwan \\ 807 \\ ${ }^{b}$ Faculty of Medicinal \& Applied Chemistry, Kaohsiung Medical University, Kaohsiung City, \\ Taiwan 807 \\ E-mail: enchwa@kmu.edu.tw
}

\begin{abstract}
The syntheses of 4-substituted and 5-substituted 2,5-dihydro-1-naphthoxepines are described. 1Naphthol, starting material, was subjected to sequential reactions of $O$-alkylation, the Wittig reaction, the Claisen reaction, $O$-allylation, and ring-closing metathesis to provide 4-substituted 2,5-dihydro-1-naphthoxepines. Similarly, 5-substituted 2,5-dihydro-1-naphthoxepines were produced in good yields.
\end{abstract}

Keywords: Ring-closing metathesis, Claisen rearrangement, 1-naphthol, 4-substituted 2,5dihydro-1-naphthoxepines, 5-substituted 2,5-dihydro-1-naphthoxepines.

\section{Introduction}

In the last decade, considerale efforts have been made in our laboratory to utilize the Claisen rearrangement and ring-closing metathesis as key steps to prepare benzocarbocyclic and benzoheterocyclic compounds. ${ }^{1}$ Recently Kotha, et al., ${ }^{2}$ using the same strategy but developing a new approach to biologically relevant 2-naphthoxepines prompted us to search for some related compounds. From a literature survey, it was clear that naphthoxepines have been paid little attention $^{3}$ and the synthesis of substituted 2,5-dihydro-1-naphthoxepines (2,5-dihydronaphth[1,2$b$ ]oxepines) have not been described. Herein, we disclose an alternative method for the synthesis of 4-substituted and 5-substituted 2,5-dihydro-1-naphthoxepines. The synthesis started from 1naphthol and was based on the Claisen rearrangement and ring-closing metathesis as key steps as shown in Schemes 1, and 2. 
Scheme 1. Synthesis of 4-substituted 2,5-dihydro-1-naphthoxepines(7a-c)
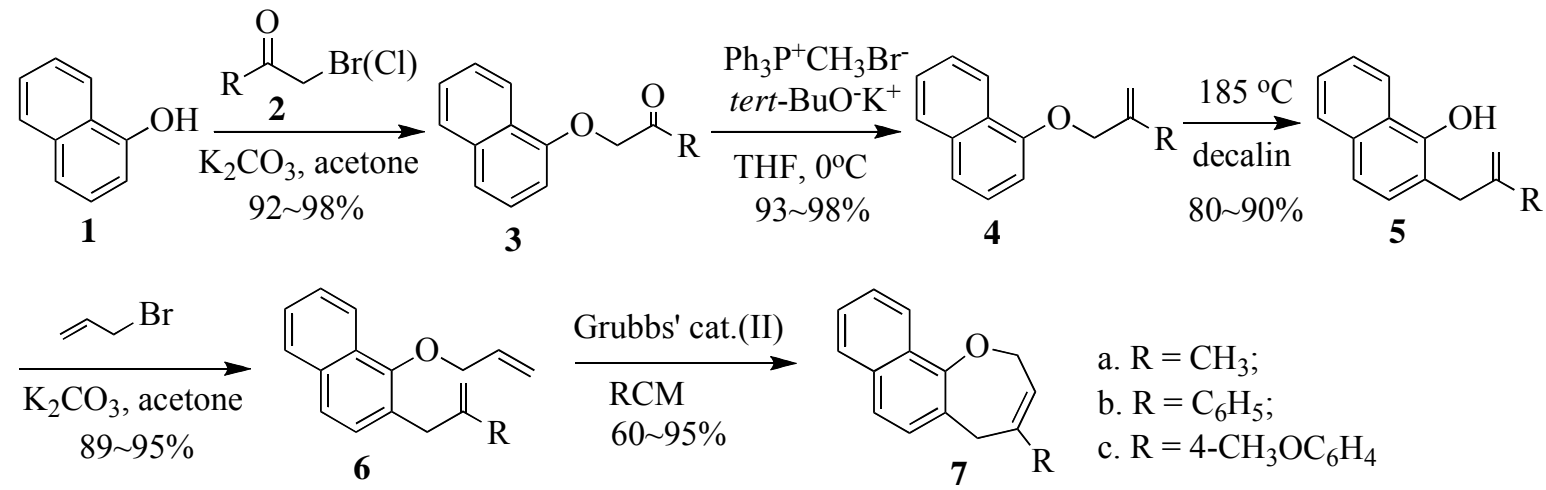

Scheme 2. Synthesis of 5-substituted 2,5-dihydro-1-naphthoxepines(12a-b)<smiles>Oc1cccc2ccccc12</smiles>

1

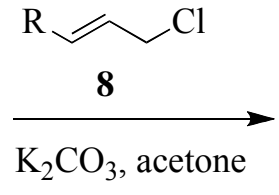
90 92\%<smiles>[R]C=CCOc1cccc2ccccc12</smiles>

9

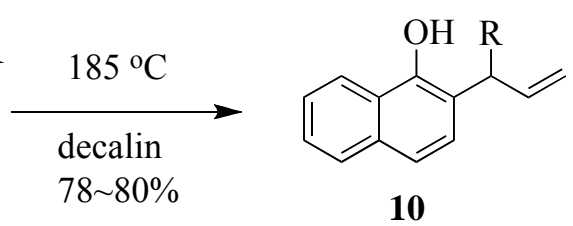

10<smiles>[R]C(C=C)c1ccc2ccccc2c1OCC=C</smiles>

a. $\mathrm{R}=\mathrm{CH}_{3} ;$ b. $\mathrm{R}=\mathrm{C}_{6} \mathrm{H}_{5}$

\section{Results and Discussion}

As shown in Scheme 1, the reaction of 1-naphthol (1) with chloroacetone (2a), bromoacetophenone (2b), and 2-bromo-1-(4-methoxyphenyl)ethanone (2c) in the presence of dry potassium carbonate in refluxing acetone for 3-4 $\mathrm{h}$, gave 1-(substituted)-2-(1naphthalenyloxy)ethanones (3a-c) in $92-98 \%$ yields. Reaction of 3a-c with methylenetriphenylphosphorane generated from the reaction of methyltriphenylphosphonium bromide and potassium tert-butoxide at $0^{\circ} \mathrm{C}$ in situ afforded 1-[2-(substituted)allyloxy]naphthalenes (4a-c) in yields of 93 - 98\%. Subsequently, compounds 4a-c were heated to $185{ }^{\circ} \mathrm{C}$ to bring about Claisen rearrangement to lead 2-(2-substituted allyl)-1-naphthols (5a-c) which have satisfactory spectral data, in yields of $80-90 \%$. The $O$-allylation of 5a-c was easily achieved by the general procedure to give 1-allyloxy-2-[2-(substituted)allyl]naphthalenes (6a-c) 
in yields of $89-95 \%$. Finally by treatment of 6a-c with Grubbs' catalyst (II) the desired 4(substituted)-2,5-dihydro-1-naphthoxepines (7a-c) were produced in yields of $60-95 \%$, respectively. Furthermore, as shown in Scheme 2, 1-allyloxynaphthalenes (9a-b) prepared from 1-naphthol (1) with crotyl chloride (8a), and cinnamyl chloride $(\mathbf{8 b})$ were heated to $185-190{ }^{\circ} \mathrm{C}$ in decalin for $0.75-2.5 \mathrm{~h}$ to give, via Claisen rearrangement, 2-allyl-1-naphthols (10a-b) in 78 $80 \%$ yields. In the reaction producing $\mathbf{9 b}$, besides the ortho-product $\mathbf{1 0 b}$, its isomeric paraproduct 10c was also obtained. In a search for the optimal conditions for yielding 10b, various conditions were investigated and the results were depicted as Table 1.

Table 1. Conditions and percentage yields of the Claisen rearrangement of $\mathbf{9 b}$

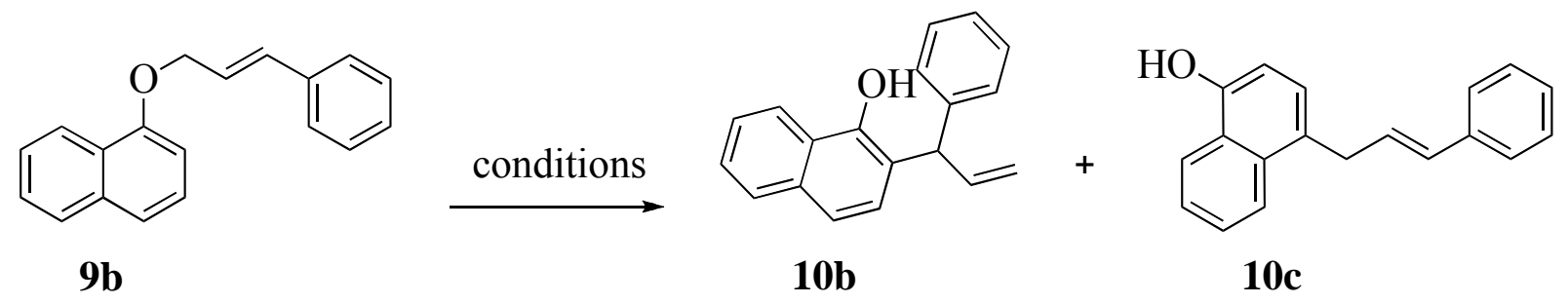

\begin{tabular}{cclll}
\hline Compound & $\begin{array}{l}\text { Conditions } \\
\left({ }^{\circ} \mathrm{C} / \text { solvent }\right)\end{array}$ & $\begin{array}{l}\text { Reaction } \\
\text { time }(\mathrm{hr})\end{array}$ & \multicolumn{2}{l}{ Products $(\% \text { yields })^{*}$} \\
\hline \multirow{2}{*}{$9 \mathbf{b}$} & 185/Decalin & 0.5 & $\mathbf{1 0 b}(51)$ & $\mathbf{1 0 c}(7)$ \\
& & 0.75 & $\mathbf{1 0 b}(78)$ & $\mathbf{1 0 c}(12)$ \\
& & 1.0 & $\mathbf{1 0 b}(65)$ & $\mathbf{1 0 c}(17)$ \\
$9 \mathbf{9 b}$ & & 1.5 & $\mathbf{1 0 b}(46)$ & $\mathbf{1 0 c}(26)$ \\
& 217/Diethylaniline & 0.5 & $\mathbf{1 0 b}(15)$ & $\mathbf{1 0 c}(68)$ \\
& & 0.75 & $\mathbf{1 0 b}(18)$ & $\mathbf{1 0 c}(48)$ \\
& & 1.0 & $\mathbf{1 0 b}(12)$ & $\mathbf{1 0 c}(63)$ \\
\end{tabular}

*The isolated yield was indicated.

As shown in Table 1, a bulky allyl group, as in $\mathbf{9 b}$, favors ortho-product $\mathbf{1 0 b}$ at shorter reaction times of $0.75 \mathrm{~h}$ in decalin. On the other hand, at the longer $1 \mathrm{~h}$ reaction time in diethylaniline, the para-product 10c predominates. This means that 10c is thermodynamically more stable than 10b because of conjugated character of 10c. The isomeric 10b and 10c can be easily distinguished by ${ }^{1} \mathrm{H}-\mathrm{NMR}$ measurements. Following the general procedure, 10a-b were reacted with allyl bromide to afford 2-allyl-1-allyloxynaphthalenes (11a-b) in 91- 92\% yields, respectively. Treatment of 11a-b with Grubbs' catalyst (II) produced 5-substituted 2,5-dihydro-1naphthoxepines (12a-b) in yields of 55 - 60\%. The compounds in Scheme 2 are all new and gave satisfactory spectral data. A comparison of selected protons for compounds 7 and 12 in ${ }^{1} \mathrm{H}-\mathrm{NMR}$ spectra is compiled at Table 2 . 
Thus, we have established a new route to prepare 4-substituted-2,5-dihydro-1naphthoxepines (7a-c) and 5-substituted 2,5-dihydro-1-naphthoxepines (12a-b), from 1naphthol.

Table 2. A comprasion of selected protons of compound 7 and 12 in ${ }^{1} \mathrm{H}-\mathrm{NMR}$
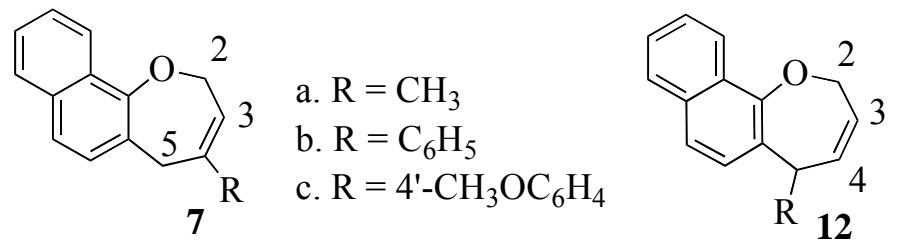
a. $\mathrm{R}=\mathrm{CH}_{3}$
b. $\mathrm{R}=\mathrm{C}_{6} \mathrm{H}_{5}$

\begin{tabular}{cccccc}
\hline Compound & H-2 & H-3 & H-4 & H-5 & Yield (\%) \\
\hline 7a & $4.63(\mathrm{dd})$ & $5.30(\mathrm{tq})$ & - & $3.55(\mathrm{~s})$ & 95 \\
7b & $4.93(\mathrm{dd})$ & $5.79(\mathrm{t})$ & - & $4.13(\mathrm{~s})$ & 60 \\
7c & $4.88(\mathrm{dd})$ & $5.71(\mathrm{t})$ & - & $4.06(\mathrm{~s})$ & 60 \\
& & & & & \\
12a & $4.54-4.76(\mathrm{~m})$ & $5.48-5.52(\mathrm{~m})$ & $5.84-5.90(\mathrm{~m})$ & - & 60 \\
12b & $4.86-4.92(\mathrm{~m})$ & $5.77-5.82(\mathrm{~m})$ & $6.10-6.16(\mathrm{~m})$ & - & 55 \\
\hline
\end{tabular}

\section{Experimental Section}

General Procedures. Melting points (Yanaco micro melting-point apparatus) are uncorrected. ${ }^{1} \mathrm{H}-\mathrm{NMR}$ and ${ }^{13} \mathrm{C}-\mathrm{NMR}$ spectra were obtained on a Varian Gemini-200 or Varian Unity plus 400 Spectrometer. Chemical shifts are indicated in parts per million with respect to TMS. Elemental analyses were recorded on a Heraeus CHN-O Rapid analyzer. Mass spectra were recorded on a Chem/hp/middle spectrometer connected to a Hewlett Packard series II model gas-liquid chromatograph. HRMS spectra were performed on a JEOL JMS SX/SX 102A instrument. Silica gel (230-400 mesh) for column chromatography and precoated silica gel plates (60 F-254) for TLC was purchased from E. Merck Co. UV light $(254 \mathrm{~nm})$ was used to detect spots on TLC plates after development.

\section{General procedure for the preparation of 1-(substituted)-2-(1-naphthalenyloxy)etha- nones (3a-c)}

Under the protection of nitrogen, to a solution of 1-naphthol $(7.21 \mathrm{~g}, 50.0 \mathrm{mmol})$ dissolved in dry acetone $(150 \mathrm{~mL})$ was added $\mathrm{K}_{2} \mathrm{CO}_{3}(9.67 \mathrm{~g}, 70.0 \mathrm{mmol})$ and substituted 2-bromoacetophenone (2a-c) $(60.0 \mathrm{mmol})$ in sequence. The reaction mixture obtained was heated to reflux for 3-4 $\mathrm{h}$, monitored by TLC. After cooling to room temperature, the resulting reaction mixture was filtered to remove the solid. The filtrate was was concentrated in vacuo to remove the solvent. 
The resulting residue was purified by column chromatography (ethyl acetate: $n$-hexane $=1: 10$ ) to provide pure 3a-c, respectively.

1-(1-Naphthalenyloxy)propan-2-one (3a). ${ }^{4}(9.40 \mathrm{~g}, 94 \%)$ was obtained as colorless liquid, $\mathrm{R}_{f}=$ 0.54 (ethyl acetate: $n$-hexane $=1: 7),{ }^{1} \mathrm{H}-\mathrm{NMR}\left(\mathrm{CDCl}_{3}, 400 \mathrm{MHz}\right) \delta 2.29\left(\mathrm{~s}, 3 \mathrm{H}, \mathrm{CH}_{3}\right), 4.56(\mathrm{~s}$, $\left.2 \mathrm{H}, \mathrm{OC}_{2} \mathrm{COCH}_{3}\right), 6.57(\mathrm{~d}, J=7.2 \mathrm{~Hz}, 1 \mathrm{H}, \mathrm{ArH}), 7.28(\mathrm{t}, J=8.4 \mathrm{~Hz}, 1 \mathrm{H}, \mathrm{ArH}), 7.43(\mathrm{~d}, J=8.4$ $\mathrm{Hz}, 1 \mathrm{H}, \mathrm{ArH}), 7.45-7.50$ (m, 2H, ArH), 7.75-7.79 (m, 1H, ArH), 8.28-8.32 (m, $1 \mathrm{H}, \mathrm{ArH}){ }^{13} \mathrm{C}-$ NMR $\left(\mathrm{CDCl}_{3}, 100 \mathrm{MHz}\right) \delta 26.6,72.9,104.7,121.2,121.7,125.2,125.4,125.5,126.5,127.4$, 134.4, 153.2, 205.6 ; IR (neat) $\mathrm{cm}^{-1}: 1110.2,1272.5,1398.1,1579.2,1721.5,2902.3,3054.5$; EIMS (70eV) m/z (rel. intensity, \%) $200\left(\mathrm{M}^{+}, 100\right), 201$ (28), 183 (15), 157 (35), 143 (16), 129 (35), 128 (18), 127 (29), 126 (13), 115 (35); HRMS (EI, m/z): Calcd. for $\mathrm{C}_{13} \mathrm{H}_{12} \mathrm{O}_{2}$ : 200.0837. Found: 200.0838.

2-(1-Naphthalenyloxy)-1-phenylethanone (3b)..$^{5}$ (12.84 g, 98\%) was obtained as colorless crystals, mp 70-71 ${ }^{\circ} \mathrm{C}, \mathrm{R}_{f}=0.63$ (ethyl acetate: $n$-hexane $\left.=1: 7\right),{ }^{1} \mathrm{H}-\mathrm{NMR}\left(\mathrm{CDCl}_{3}, 200 \mathrm{MHz}\right) \delta$ $5.41\left(\mathrm{~s}, 2 \mathrm{H}, \mathrm{ArOC}_{2} \mathrm{CC}_{6} \mathrm{H}_{5}\right), 6.78(\mathrm{~d}, J=7.6 \mathrm{~Hz}, 1 \mathrm{H}, \mathrm{ArH}), 7.35$ (t, J=8.2 Hz, 1H, ArH), 7.47$7.56\left(\mathrm{~m}, 2 \mathrm{H}, \mathrm{ArOCH}_{2} \mathrm{CC}_{6} \underline{\mathrm{H}}_{5}\right), 7.58-7.66\left(\mathrm{~m}, 4 \mathrm{H}, \mathrm{ArH}, \mathrm{ArOCH}_{2} \mathrm{C}-\mathrm{C}_{6} \underline{\mathrm{H}}_{5}\right), 7.80-7.85(\mathrm{~m}, 1 \mathrm{H}$, $\mathrm{ArH}), 8.04-8.09$ (m, 2H, $\left.\mathrm{ArOCH}_{2} \mathrm{CC}_{6} \underline{\mathrm{H}}_{5}\right), 8.37-8.42(\mathrm{~m}, 1 \mathrm{H}, \mathrm{ArH}) ;{ }^{13} \mathrm{C}-\mathrm{NMR}\left(\mathrm{CDCl}_{3}, 50 \mathrm{MHz}\right)$ $\delta$ 71.2, 105.3, 121.3, 122.2, 125.5, 125.6, 126.6, 127.4, 128.3, 128.8, 133.8, 134.6, 153.8, 154.9, 194.5 ; IR (KBr) cm $\mathrm{cm}^{-1}: 1122.2,1217.9,1395.4,1579.3,1704.6,2902.9,3059.7$; EI-MS (70eV) m/z (rel. intensity,\%) $262\left(\mathrm{M}^{+}, 35\right), 128$ (10), 127 (24), 126(10), 115(27), 106 (8), 105 (100), 91 (14), 77 (52), 51 (16); HRMS (EI, m/z): Calcd. for $\mathrm{C}_{18} \mathrm{H}_{14} \mathrm{O}_{2}$ : 262.0994. Found: 262.0993.

1-(4-Methoxyphenyl)-2-(1-naphthalenyloxy)ethanone (3c). ${ }^{6}(13.49 \mathrm{~g}, 92 \%)$ was obtained as colorless crystals, mp $77-78{ }^{\circ} \mathrm{C}, \mathrm{R}_{f}=0.47$ (ethyl acetate: $n$-hexane $\left.=1: 7\right),{ }^{1} \mathrm{H}-\mathrm{NMR}\left(\mathrm{CDCl}_{3}, 400\right.$ $\mathrm{MHz}) \delta 3.86\left(\mathrm{~s}, 3 \mathrm{H}, \mathrm{OCH}_{3}\right), 5.34\left(\mathrm{~s}, 2 \mathrm{H}, \mathrm{ArOCH}_{2} \mathrm{CO}\right), 6.77(\mathrm{~d}, J=7.6 \mathrm{~Hz}, 1 \mathrm{H}, \mathrm{ArH}), 6.95(\mathrm{dt}, J$ =9.6, $2.8 \mathrm{~Hz}, 2 \mathrm{H}, \mathrm{ArH}), 7.33(\mathrm{t}, J=8.0 \mathrm{~Hz}, 1 \mathrm{H}, \mathrm{ArH}), 7.44-7.52(\mathrm{~m}, 3 \mathrm{H}, \mathrm{ArH}), 7.79$ (dt, $J=6.8$, $2.4 \mathrm{~Hz}, 1 \mathrm{H}, \mathrm{ArH}), 8.05$ (dt, $J=9.6,2.8 \mathrm{~Hz}, 2 \mathrm{H}, \mathrm{ArH}), 8.34-8.36$ (m, $1 \mathrm{H}, \mathrm{ArH}) ;{ }^{13} \mathrm{C}-\mathrm{NMR}$ $\left(\mathrm{CDCl}_{3}, 100 \mathrm{MHz}\right) \delta 55.5,71.2,105.2,114.0,121.2,122.1,125.4,125.6,126.5,127.4,127.7$, 130.7, 134.5, 153.8, 164.0, 193.2; IR (KBr) cm $\mathrm{cm}^{-1}: 1121.9,1178.7,1228.4,1395.2,1598.0,1687.5$, 2913.2, 3052.2 ; EI-MS (70 eV) m/z (rel. intensity, \%): $292\left(\mathrm{M}^{+}, 68\right), 149$ (7), 136 (24), 135 (100), 121(14), 115 (8), 77 (17), HRMS (EI, m/z): Calcd. for $\mathrm{C}_{19} \mathrm{H}_{16} \mathrm{O}_{3}$ : 292.1099. Found: 292.1120 .

\section{General procedure for the preparation of 1-[2-(substituted)allyloxy]naphthalene (4a-c)}

Under dry nitrogen, methyltriphenylphosphonium bromide $(10.72 \mathrm{~g}, 30.0 \mathrm{mmol})$ suspended in dry THF $(100 \mathrm{~mL})$ was cooled to $0^{\circ} \mathrm{C}$. To this cooled suspension, $t-\mathrm{BuO}^{-} \mathrm{K}^{+}(3.64 \mathrm{~g}, 32.5 \mathrm{mmol})$ was added in portions and the mixture was stirred at $0{ }^{\circ} \mathrm{C}$ for $30 \mathrm{~min}$. After which time, 1substituted-2-(1-naphthalenyloxy)ethanone (3a-c) $(25.0 \mathrm{mmol})$ in anhydrous THF ( $50 \mathrm{~mL})$ was added, and the mixture was left stirring for $3 \mathrm{~h}$ at $0{ }^{\circ} \mathrm{C}$. Then, the resulting mixture was quenched with saturated $\mathrm{NH}_{4} \mathrm{Cl}$ solution and extracted with dichloromethane $(50 \mathrm{~mL} \times 3)$. The organic layers were combined, washed with brine, and then dried with anhydrous magnesium sulfate. After filtration, the filtrate was concentrated in vacuo to remove the solvent. The 
resulting residue was purified by silica gel column chromatography (ethyl acetate: $n$-hexane $=1$ : 10) to give pure 4a-e, respectively.

1-(2-Methyallyloxy)naphthalene (4a). ${ }^{7}$ (4.85 g, 98\%) was obtained as colorless liquid, $\mathrm{R}_{f}=0.89$ (ethyl acetate: $n$-hexane $=1: 7) ;{ }^{1} \mathrm{H}-\mathrm{NMR}\left(\mathrm{CDCl}_{3}, 400 \mathrm{MHz}\right) \delta 1.88\left(\mathrm{~s}, 3 \mathrm{H}, \mathrm{CH}_{3}\right), 4.55(\mathrm{~s}, 2 \mathrm{H}$, $\left.\mathrm{OC}_{2} \mathrm{COCH}_{3}\right), 5.02\left(\mathrm{~d}, J=1.2 \mathrm{~Hz}, 1 \mathrm{H}, \mathrm{OCH}_{2} \mathrm{C}=\mathrm{C}_{\mathrm{H}_{\mathrm{a}}} \mathrm{H}_{\mathrm{b}}\right), 5.19(\mathrm{~d}, J=1.2 \mathrm{~Hz}, 1 \mathrm{H}$, $\left.\mathrm{OCH}_{2} \mathrm{C}=\mathrm{CH}_{\mathrm{a}} \underline{\mathrm{H}}_{\mathrm{b}}\right), 6.75(\mathrm{~d}, J=7.6 \mathrm{~Hz}, 1 \mathrm{H}, \mathrm{ArH}), 7.31(\mathrm{t}, J=8.0 \mathrm{~Hz}, 1 \mathrm{H}, \mathrm{ArH}), 7.39(\mathrm{~d}, J=8.4$ $\mathrm{Hz}, 1 \mathrm{H}, \mathrm{ArH}), 7.43-7.47$ (m, 2H, ArH), 7.74-7.81 (m, 1H, ArH), 8.30-8.33 (m, 1H, ArH); ${ }^{13} \mathrm{C}-$ NMR $\left(\mathrm{CDCl}_{3}, 100 \mathrm{MHz}\right) \delta$ 19.6, 71.8, 105.0, 112.6, 120.3, 122.1, 125.2, 125.8, 125.8, 126.4, 127.5, 134.5, 140.9, 154.4; EI-MS (70eV) m/z (rel. intensity, \%) $198\left(\mathrm{M}^{+}, 100\right), 199$ (18), 183(59), 165 (16), 157 (9), 156 (11), 155 (32), 153 (15), 129 (14), 128 (22); HRMS (EI, m/z): Calcd. for $\mathrm{C}_{14} \mathrm{H}_{14} \mathrm{O}$ : 198.1045 . Found: 198.1048 .

1-(2-Phenylallyloxy)naphthalene (4b). ${ }^{8}(6.32 \mathrm{~g}, 98 \%)$ was obtained as colorless liquid, $\mathrm{R}_{f}=$ 0.83 (ethyl acetate: $n$-hexane $=1: 7),{ }^{1} \mathrm{H}-\mathrm{NMR}\left(\mathrm{CDCl}_{3}, 400 \mathrm{MHz}\right) \delta 4.91(\mathrm{~s}, 2 \mathrm{H}$, $\left.\operatorname{ArOC} \underline{H}_{2} \mathrm{CC}_{6} \mathrm{H}_{5}\right), 5.50\left(\mathrm{~d}, J=0.8 \mathrm{~Hz}, 1 \mathrm{H}, \operatorname{ArOCH}_{2} \mathrm{C}=\mathrm{C}_{\mathrm{H}_{a}} \mathrm{H}_{\mathrm{b}}\right), 5.58(\mathrm{~d}, J=0.8 \mathrm{~Hz}, 1 \mathrm{H}$, $\left.\operatorname{ArOCH}_{2} \mathrm{C}=\mathrm{CH}_{\mathrm{a}} \underline{\mathrm{H}}_{\mathrm{b}}\right), 6.75(\mathrm{~d}, J=8.0 \mathrm{~Hz}, 1 \mathrm{H}, \mathrm{ArH}), 7.20-7.45\left(\mathrm{~m}, 9 \mathrm{H}, \mathrm{ArH}, \mathrm{ArOCH}_{2} \mathrm{C}-\mathrm{C}_{6} \underline{\mathrm{H}}_{5}\right)$, 7.70-7.72 (m, 1H, ArH), 8.23-8.25 (m, 1H, $\mathrm{ArH}) ;{ }^{13} \mathrm{C}-\mathrm{NMR}\left(\mathrm{CDCl}_{3}, 100 \mathrm{MHz}\right) \delta$ 69.8, 105.1, $114.6,120.5,122.1,125.2,125.7,126.0,126.4,127.4,127.9,128.4,134.5,138.3,142.9,154.2$; EI-MS (70eV) m/z (rel. intensity,\%) 260 (M+, 100), 261 (21), 259 (27), 246 (11), 245 (47), 217 (27), 215 (12), 129 (22), 128 (29), 77 (14); HRMS (EI, m/z): Calcd. for $\mathrm{C}_{19} \mathrm{H}_{16} \mathrm{O}: 260.1201$. Found: 260.1205.

1-[2-(4-Methoxyphenyl)allyloxy]naphthalene (4c) (6.79 g, 93\%) was obtained as colorless liquid, $\mathrm{R}_{f}=0.72$ (ethyl acetate: $n$-hexane $\left.=1: 7\right),{ }^{1} \mathrm{H}-\mathrm{NMR}\left(\mathrm{CDCl}_{3}, 200 \mathrm{MHz}\right) \delta 3.71(\mathrm{~s}, 3 \mathrm{H}$, $\left.\mathrm{OCH}_{3}\right), 4.96\left(\mathrm{~s}, 2 \mathrm{H}, \operatorname{ArOC}_{2}\right), 5.45\left(\mathrm{~d}, J=1.2 \mathrm{~Hz}, 1 \mathrm{H}, \mathrm{R}_{1} \mathrm{R}_{2} \mathrm{C}=\mathrm{C}_{3} \mathrm{H}_{\mathrm{b}}\right), 5.96,(\mathrm{~d}, J=1.2 \mathrm{~Hz}, 1 \mathrm{H}$, $\mathrm{R}_{1} \mathrm{R}_{2} \mathrm{C}=\mathrm{CH}_{\mathrm{a}} \underline{\mathrm{H}}_{\mathrm{b}}$ ), 6.78-6.86 (m, 3H, ArH), 7.28-7.46 (m, 6H, ArH), 7.73-7.77 (m, 1H, ArH), 8.21$8.26(\mathrm{~m}, 1 \mathrm{H}, \mathrm{ArH}),{ }^{13} \mathrm{C}-\mathrm{NMR}\left(\mathrm{CDCl}_{3}, 50 \mathrm{MHz}\right) \delta 55.1,70.0,105.1,113.0,113.7,120.4,122.1$, 125.2, 125.7, 126.3, 127.1, 127.3, 130.7, 134.5, 142.2, 154.3, 159.4; EI-MS (70 eV) m/z (rel. intensity, \%): $290\left(\mathrm{M}^{+}, 100\right), 276$ (12), 275 (6), 274 (15), 247 (5), 246 (7), HRMS (EI, m/z): Calcd. for $\mathrm{C}_{20} \mathrm{H}_{18} \mathrm{O}_{2}: 290.1307$. Found: 290.1285 .

\section{General procedure for the preparation of 2-(2-substituted allyl)-1-naphthol (5a-c)}

Under the protection of dry nitrogen, 1-(2-substituted allyloxy)naphthalene (4a-c) (23.0 mmol) in decalin $(30 \mathrm{~mL})$ was heated to $185-190{ }^{\circ} \mathrm{C}$ for $2.5 \mathrm{~h}$. The reaction mixture was distilled off the solvent by Kugehror apparatus at $4 \mathrm{~mm} \mathrm{Hg}$. The resulting residue was purified by silica gel column chromatography (ethyl acetate: $n$-hexane $=1: 10$ ) to give pure 5a-c, respectively.

2-(2-Methylallyl)-1-naphthol (5a). ${ }^{7}\left(3.97 \mathrm{~g}, 87 \%\right.$ ) was obtained as colorless liquid, $\mathrm{R}_{f}=0.58$ (ethyl acetate: $n$-hexane $=1: 7),{ }^{1} \mathrm{H}-\mathrm{NMR}\left(\mathrm{CDCl}_{3}, 400 \mathrm{MHz}\right) \delta 1.75\left(\mathrm{~s}, 3 \mathrm{H}, \mathrm{CH}_{3}\right), 3.53(\mathrm{~s}, 2 \mathrm{H}$, $\left.\mathrm{ArCH}_{2} \mathrm{C}=\mathrm{CH}_{2}\right), 4.98\left(\mathrm{~d}, J=1.2 \mathrm{~Hz}, 1 \mathrm{H}, \mathrm{ArCH}_{2} \mathrm{C}=\mathrm{CH}_{\mathrm{a}} \mathrm{H}_{\mathrm{b}}\right), 4.99\left(\mathrm{~d}, J=1.2 \mathrm{~Hz}, 1 \mathrm{H}, \mathrm{ArCH}_{2} \mathrm{C}=\right.$ $\left.\mathrm{CH}_{\mathrm{a}} \underline{\mathrm{H}}_{\mathrm{b}}\right), 5.77(\mathrm{~s}, 1 \mathrm{H}, \mathrm{OH}), 7.20(\mathrm{~d}, J=8.8 \mathrm{~Hz}, 1 \mathrm{H}, \mathrm{ArH}), 7.39(\mathrm{~d}, J=8.4 \mathrm{~Hz}, 1 \mathrm{H}, \mathrm{ArH}), 7.42-$ $7.48(\mathrm{~m}, 2 \mathrm{H}, \mathrm{ArH}), 7.76-7.78(\mathrm{~m}, 1 \mathrm{H}, \mathrm{ArH}), 8.17-8.20(\mathrm{~m}, 1 \mathrm{H}, \mathrm{ArH}) ;{ }^{13} \mathrm{C}-\mathrm{NMR}\left(\mathrm{CDCl}_{3}, 100\right.$ $\mathrm{MHz}) \delta 22.0,40.7,112.8,117.6,120.1,121.5,124.9,125.2,125.8,127.4,129.0,133.8,144.7$, 
150.1 ; EI-MS (70eV) m/z (rel. intensity,\%) $198\left(\mathrm{M}^{+}, 100\right), 199$ (16), 183 (77), 165 (26), 156 (16), 155 (50), 153 (25), 152 (13), 129 (23), 128 (44); HRMS (EI, m/z): Calcd. for $\mathrm{C}_{14} \mathrm{H}_{14} \mathrm{O}$ : 198.1045. Found: 198.1043.

2-(2-Phenylallyl)-1-naphthol (5b). (5.38 g, 90\%) was obtained as colorless crystals, mp 114$115{ }^{\circ} \mathrm{C}, \mathrm{R}_{f}=0.66$ (ethyl acetate: $n$-hexane $\left.=1: 7\right) ;{ }^{1} \mathrm{H}-\mathrm{NMR}\left(\mathrm{CDCl}_{3}, 400 \mathrm{MHz}\right) \delta 3.95(\mathrm{~s}, 2 \mathrm{H}$, $\left.\mathrm{ArC}_{2} \mathrm{C}=\mathrm{CH}_{2}\right), 5.08(\mathrm{~s}, 1 \mathrm{H}, \mathrm{ArOH}), 5.53\left(\mathrm{~d}, J=0.8 \mathrm{~Hz}, 1 \mathrm{H}, \mathrm{ArCH}_{2} \mathrm{C}=\mathrm{C}_{\mathrm{H}} \mathrm{H}_{\mathrm{b}}\right), 5.57(\mathrm{~d}, J=0.8$ $\left.\mathrm{Hz}, 1 \mathrm{H}, \quad \mathrm{ArCH}_{2} \mathrm{C}=\mathrm{CH}_{\mathrm{a}} \underline{\mathrm{H}}_{\mathrm{b}}\right), 7.23(\mathrm{~d}, J=8.0 \mathrm{~Hz}, 1 \mathrm{H}, \operatorname{ArH}), 7.25-7.31(\mathrm{~m}, 3 \mathrm{H}, \operatorname{ArH}$, $\left.\mathrm{ArCH}_{2} \mathrm{CC}_{6} \underline{\mathrm{H}}_{5}\right), 7.37$ (d, $\left.J=8.4 \mathrm{~Hz}, 1 \mathrm{H}, \mathrm{ArH}\right), 7.39-7.48$ (m, 4H, ArH, $\left.\mathrm{ArCH}_{2} \mathrm{CC}_{6} \underline{\mathrm{H}}_{5}\right), 7.73-7.75$ $(\mathrm{m}, 1 \mathrm{H}, \mathrm{ArH}), 8.13-8.16(\mathrm{~m}, 1 \mathrm{H}, \mathrm{ArH}) ;{ }^{13} \mathrm{C}-\mathrm{NMR}\left(\mathrm{CDCl}_{3}, 100 \mathrm{MHz}\right) \delta 37.0,114.3,117.9$, $120.4,121.3,124.8,125.2,125.8,126.0,127.5,127.9,128.4,128.8,133.7,140.1,145.8,149.5$; EI-MS (70eV) m/z (rel. intensity,\%) $260\left(\mathrm{M}^{+}, 100\right), 261$ (21), 259 (28), 245 (51), 217 (34), 215 (17), 129 (34), 128 (54), 127 (19), 77 (20); HRMS (EI, m/z): Calcd. for $\mathrm{C}_{19} \mathrm{H}_{16} \mathrm{O}: 260.1201$, Found: 260.1204.

2-[2-(4-Methoxyphenyl)allyl]-1-naphthol (5c). (5.34 g, 80\%) was obtained as colorless crystals, mp $123-124{ }^{\circ} \mathrm{C}, \mathrm{R}_{f}=0.53$ (ethyl acetate: $n$-hexane $\left.=1: 7\right),{ }^{1} \mathrm{H}-\mathrm{NMR}\left(\mathrm{CDCl}_{3}, 400 \mathrm{MHz}\right)$

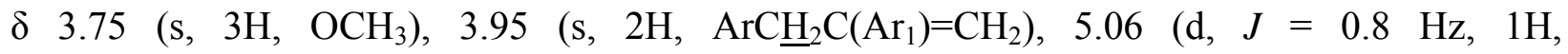
$\left.\mathrm{ArCH}_{2} \mathrm{C}\left(\mathrm{Ar}_{1}\right)=\mathrm{C}_{\mathrm{H}} \mathrm{H}_{\mathrm{b}}\right), 5.48\left(\mathrm{~d}, J=0.8 \mathrm{~Hz}, 1 \mathrm{H}, \mathrm{ArCH}_{2} \mathrm{C}\left(\mathrm{Ar}_{1}\right)=\mathrm{CH}_{a} \underline{\mathrm{H}}_{\mathrm{b}}\right), 5.65(\mathrm{~s}, 1 \mathrm{H}, A r O H)$, $6.82(\mathrm{dt}, J=9.6,2.8 \mathrm{~Hz}, 2 \mathrm{H}, \mathrm{ArH}), 7.22(\mathrm{~d}, J=8.0 \mathrm{~Hz}, 1 \mathrm{H}, \mathrm{ArH}), 7.38(\mathrm{~d}, J=8.0 \mathrm{~Hz}, 1 \mathrm{H}, \mathrm{ArH})$, 7.39-7.46 (m, 4H, ArH), 7.74-7.76 (m, 1H, ArH), 8.14-8.16 (m, 1H, ArH), ${ }^{13} \mathrm{C}-\mathrm{NMR}\left(\mathrm{CDCl}_{3}\right.$, $100 \mathrm{MHz}) \delta 37.3,55.2,112.7,113.7,117.9,120.3,121.4,124.8,125.2,125.7,127.2,127.5$, 128.9, 132.3, 133.7, 145.2, 149.7, 159.4; EI-MS (70 eV) m/z (rel. intensity, \%): $290\left(\mathrm{M}^{+}, 100\right)$, 276 (11), 275 (52), 247 (23), 215 (10), 202 (10), 181(14), 128 (13); HRMS (EI, m/z): Calcd. for $\mathrm{C}_{20} \mathrm{H}_{18} \mathrm{O}_{2}$ : 290.1307. Found: 290.1285 .

General procedure for the preparation of 1-allyloxy-2-[2-(substituted)allyl]naphtha- lene (6a-c)

Under the protection of dry nitrogen, to 2-[2-(substituted)allyl]-1-naphthol $20.0 \mathrm{mmol}$ (5a-c) dissolved in dry acetone $(120 \mathrm{~mL})$ was added $\mathrm{K}_{2} \mathrm{CO}_{3}(3.87 \mathrm{~g}, 28.0 \mathrm{mmol})$ and allyl bromide $(2.90 \mathrm{~g}, 24.0 \mathrm{mmol})$. The reaction mixture was heated at reflux for $4 \mathrm{~h}$. After cooling to room temperature, the reaction mixture was filtered to remove the solid. The filtrate which was obtained was concentrated in vacuo to remove the solvent. The residue which was obtained was purified by column chromatography (ethyl acetate: $n$-hexane $=1: 10$ ) to give pure 6a-c, respectively.

1-Allyloxy-2-(2-methylallyl)naphthalene (6a). (3.40 g, 95\%) was obtained as a colorless liquid, $\mathrm{R}_{f}=0.90$ (ethyl acetate: $n$-hexane $\left.=1: 7\right),{ }^{1} \mathrm{H}-\mathrm{NMR}\left(\mathrm{CDCl}_{3}, 400 \mathrm{MHz}\right) \delta 1.74\left(\mathrm{~s}, 3 \mathrm{H}, \mathrm{CH}_{3}\right), 3.53$ (s, $\left.2 \mathrm{H}, \mathrm{ArC}_{2} \mathrm{C}=\mathrm{CH}_{2}\right), 4.47$ (dt, $\left.J=5.2,1.6 \mathrm{~Hz}, 2 \mathrm{H}, \mathrm{ArOC}_{2} \mathrm{CHCH}_{2}\right), 4.68(\mathrm{~d}, J=1.2 \mathrm{~Hz}, 1 \mathrm{H}$, $\mathrm{ArCH}_{2} \mathrm{C}=\mathrm{CH}_{\mathrm{a}} \mathrm{H}_{\mathrm{b}}$ ), $4.84\left(\mathrm{~d}, J=1.2 \mathrm{~Hz}, 1 \mathrm{H}, \mathrm{ArCH}_{2} \mathrm{C}=\mathrm{CH}_{a} \underline{\mathrm{H}}_{\mathrm{b}}\right.$ ), 5.29 (ddt, $J=10.4,1.6,1.6 \mathrm{~Hz}$, $1 \mathrm{H}, \mathrm{ArOCH}_{2} \mathrm{CH}=\mathrm{C}_{\mathrm{H}} \mathrm{H} \mathrm{b}$ ), $5.50\left(\mathrm{ddt}, J=17.2,1.6,1.6 \mathrm{~Hz}, 1 \mathrm{H}, \mathrm{ArOCH}_{2} \mathrm{CH}_{=} \mathrm{CH}_{\mathrm{a}} \underline{\mathrm{H}}_{\mathrm{b}}\right), 6.18$ (ddt, $J$ $\left.=17.2,10.4,5.2 \mathrm{~Hz}, 1 \mathrm{H}, \mathrm{ArOCH}_{2} \mathrm{CH}=\mathrm{CH}_{2}\right), 7.29(\mathrm{~d}, J=8.4 \mathrm{~Hz}, 1 \mathrm{H}, \mathrm{ArH}), 7.39-7.48(\mathrm{~m}, 2 \mathrm{H}$, $\mathrm{ArH}), 7.54(\mathrm{~d}, J=8.4 \mathrm{~Hz}, 1 \mathrm{H}, \mathrm{ArH}), 7.77-7.79(\mathrm{~m}, 1 \mathrm{H}, \mathrm{ArH}), 8.08-8.10(\mathrm{~m}, 1 \mathrm{H}, \mathrm{ArH}) ;{ }^{13} \mathrm{C}-\mathrm{NMR}$ 
$\left(\mathrm{CDCl}_{3}, 100 \mathrm{MHz}\right) \delta 22.6,37.9,75.2,112.0,117.1,122.1,123.8,125.5,125.8,127.9,128.0$, 128.3, 128.4, 133.9, 134.0, 144.9, 152.6; EI-MS (70eV) m/z (rel. intensity, \%) $238\left(\mathrm{M}^{+}, 100\right)$, 239 (21), 223(21), 195(32), 182(41), 181(60), 165(50), 153(38), 152(26), 141(23); HRMS (EI, $\mathrm{m} / \mathrm{z}$ ): Calcd. for $\mathrm{C}_{17} \mathrm{H}_{18} \mathrm{O}: 238.1358$. Found: 238.1356 .

1-Allyloxy-2-(2-phenylallyl)naphthalene (6b). (5.42 g, 95\%) was obtained as a colorless liquid, $\mathrm{R}_{f}=0.86$ (ethyl acetate: $n$-hexane $\left.=1: 7\right),{ }^{1} \mathrm{H}-\mathrm{NMR}\left(\mathrm{CDCl}_{3}, 400 \mathrm{MHz}\right) \delta 4.03(\mathrm{~s}, 2 \mathrm{H}$, $\left.\operatorname{ArCH}_{2} \mathrm{C}=\mathrm{CH}_{2}\right), 4.50\left(\mathrm{dt}, J=5.2,1.6 \mathrm{~Hz}, 2 \mathrm{H}, \operatorname{ArOC}_{2}{ }_{2} \mathrm{CH}=\mathrm{CH}_{2}\right), 4.94(\mathrm{~d}, J=1.2 \mathrm{~Hz}, 1 \mathrm{H}$, $\left.\mathrm{ArCH}_{2} \mathrm{C}=\mathrm{C}_{\mathrm{H}_{\mathrm{a}}} \mathrm{H}_{\mathrm{b}}\right), 5.27$ (ddt, $\left.J=10.4,1.6,1.6, \mathrm{~Hz}, 1 \mathrm{H}, \mathrm{ArOCH}_{2} \mathrm{CH}=\mathrm{C}_{\mathrm{a}} \mathrm{H}_{\mathrm{b}}\right), 5.48$ (ddt, $J=17.2$, 1.6, $\left.1.6 \mathrm{~Hz}, 1 \mathrm{H}, \mathrm{ArOCH}_{2} \mathrm{CH}=\mathrm{CH}_{\mathrm{a}} \underline{\mathrm{H}}_{\mathrm{b}}\right), 5.49$ (d, $\left.J=1.2 \mathrm{~Hz}, 1 \mathrm{H}, \mathrm{ArCH}_{2} \mathrm{C}=\mathrm{CH}_{\mathrm{a}} \underline{\mathrm{H}}_{\mathrm{b}}\right), 6.17$ (ddt, $J=$ 17.2, 10.4, 5.2 Hz, 1H, $\left.\operatorname{ArOCH}_{2} \mathrm{C} \underline{\mathrm{H}}=\mathrm{CH}_{\mathrm{a}} \mathrm{H}_{\mathrm{b}}\right), 7.16-7.28\left(\mathrm{~m}, 3 \mathrm{H}, \mathrm{ArH}, \mathrm{ArCH}_{2} \mathrm{CC}_{6} \underline{\mathrm{H}}_{5}\right), 7.31$ (d, $J$ $=8.8 \mathrm{~Hz}, 1 \mathrm{H}, \mathrm{ArH}), 7.38-7.52\left(\mathrm{~m}, 5 \mathrm{H}, \mathrm{ArH}, \mathrm{ArCH}_{2} \mathrm{CC}_{6} \underline{\mathrm{H}}_{5}\right)$, 7.74-7.77 (m, 1H, ArH), 8.09-8.11 $(\mathrm{m}, 1 \mathrm{H}, \mathrm{ArH}) ;{ }^{13} \mathrm{C}-\mathrm{NMR}\left(\mathrm{CDCl}_{3}, 100 \mathrm{MHz}\right) \delta 35.1,75.2,114.4,117.3,122.2,123.9,125.6$, $125.8,126.0,127.5,127.8,127.9,128.2,128.3,128.4,133.9,133.9,140.9,147.0,152.5$; EI-MS $(70 \mathrm{eV}) \mathrm{m} / \mathrm{z}$ (rel.intensity,\%) $300\left(\mathrm{M}^{+}, 100\right), 301$ (24), 285 (15), 244 (16), 182 (13), 181 (26), 165 (22), 153 (17), 152 (19), 115(14); HRMS (EI, m/z): Calcd. for $\mathrm{C}_{22} \mathrm{H}_{20} \mathrm{O}$ : 300.1514, Found: 300.1516 .

1-Allyloxy-2-[2-(4-methoxyphenyl)allyl]naphthalene (6c). (5.87 g, 89\%) was obtained as a colorless liquid, $\mathrm{R}_{f}=0.75$ (ethyl acetate: $n$-hexane $\left.=1: 7\right),{ }^{1} \mathrm{H}-\mathrm{NMR}\left(\mathrm{CDCl}_{3}, 400 \mathrm{MHz}\right) \delta 3.67$ (s, $\left.3 \mathrm{H}, \mathrm{CH}_{3}\right), 3.99$ (s, 2H, $\left.\mathrm{CH}_{2}\right), 4.50$ (dt, $\left.J=5.2,1.6 \mathrm{~Hz}, 2 \mathrm{H}, \mathrm{ArOC}_{2} \mathrm{CH} \mathrm{CH}_{2}\right), 4.88(\mathrm{~d}, J=1.2$ $\left.\mathrm{Hz}, 1 \mathrm{H}, \mathrm{R}_{1} \mathrm{R}_{2} \mathrm{C}=\mathrm{C}_{\mathrm{H}} \mathrm{H}_{\mathrm{b}}\right), 5.25-5.29\left(\mathrm{~m}, 1 \mathrm{H}, \mathrm{ArOCH}_{2} \mathrm{CH}=\mathrm{C}_{\mathrm{H}_{\mathrm{a}}} \mathrm{H}_{\mathrm{b}}\right), 5.43(\mathrm{~d}, J=1.2 \mathrm{~Hz}, 1 \mathrm{H}$, $\left.\mathrm{R}_{1} \mathrm{R}_{2} \mathrm{C}=\mathrm{CH}_{\mathrm{a}} \underline{\mathrm{H}}_{\mathrm{b}}\right), 5.46-5.51\left(\mathrm{~m}, 1 \mathrm{H}, \operatorname{ArOCH}_{2} \mathrm{CH}=\mathrm{CH}_{\mathrm{a}} \underline{\mathrm{H}}_{\mathrm{b}}\right), 6.12-6.22\left(\mathrm{~m}, 1 \mathrm{H}, \operatorname{ArOCH}_{2} \mathrm{CH}=\mathrm{CH}_{2}\right)$, $6.77(\mathrm{dt}, J=6.8,2.4 \mathrm{~Hz}, 2 \mathrm{H}, \mathrm{ArH}), 7.31$ (d, $J=8.8 \mathrm{~Hz}, 1 \mathrm{H}, \mathrm{ArH}), 7.36-7.46$ (m, 4H, ArH) 7.49 $(\mathrm{d}, J=8.4 \mathrm{~Hz}, 1 \mathrm{H}, \mathrm{ArH}), 7.74(\mathrm{~d}, J=8.0 \mathrm{~Hz}, 1 \mathrm{H}, \mathrm{ArH}), 8.10(\mathrm{~d}, J=8.4 \mathrm{~Hz}, 1 \mathrm{H}, \mathrm{ArH}) ;{ }^{13} \mathrm{C}-\mathrm{NMR}$ $\left(\mathrm{CDCl}_{3}, 100 \mathrm{MHz}\right) \delta 35.1,55.0,75.1,112.7,113.5,117.3,122.1,123.9,125.5,125.8,127.0$, 127.8, 127.9, 128.2, 128.3, 133.1, 133.8, 133.9, 146.0, 152.4 159.0; EI-MS (70 eV) m/z (rel. intensity, \%): $330\left(\mathrm{M}^{+}, 100\right), 329$ (6), 316 (8), 315 (31), 289 (14), 287 (5), 274 (16), 181(8); HRMS (EI, $\mathrm{m} / \mathrm{z}$ ): Calcd. for $\mathrm{C}_{23} \mathrm{H}_{22} \mathrm{O}_{2}$ : 330.1620. Found: 330.1632 .

General procedure for the preparation of 4-substituted-2,5-dihydro-1-naphthoxepine (7a-c) 1-Allyloxy-2-[2-(substituted)allyl]naphthalene (6a-c) $(2.0 \mathrm{mmol})$ dissolved in dichloro- methane $(200 \mathrm{~mL})$ was stirred and Grubbs' catalyst (II) $(0.085 \mathrm{~g}, 5 \% \mathrm{~mol})$ added under the protection of dry nitrogen. The reaction mixture was continually stirred at room temperature (7a) for $10 \mathrm{~min}$., (7b) and (7c) for $24 \mathrm{~h}$ until the consumption of starting material as monitored by TLC. After filtration, the filtrate was concentrated in vacuo to remove the solvent. The resulting residue was purified by column chromatography (ethyl acetate: $n$-hexane $=1: 50$ ) to give pure 7a-c, respectively.

4-Methyl-2,5-dihydro-1-naphthoxepine (7a). (0.40 g, 95\%) was obtained as a colorless liquid, $\mathrm{R}_{f}=0.90$ (ethyl acetate: $n$-hexane $\left.=1: 7\right) ;{ }^{1} \mathrm{H}$-NMR $\left(\mathrm{CDCl}_{3}, 400 \mathrm{MHz}\right) \delta 1.85$ (d, $J=1.6 \mathrm{~Hz}$, $\left.3 \mathrm{H}, \mathrm{ArOCH}_{2} \mathrm{CH}=\mathrm{CRCH}_{3}\right), 3.55$ (s, 2H, $\mathrm{ArOCH}_{2} \mathrm{CH}=\mathrm{C}\left(\mathrm{CH}_{3}\right) \underline{\mathrm{C}}_{2}$ ), 4.63 (each $1 \mathrm{H}, \mathrm{dd}, J=3.6$, $\left.3.6 \mathrm{~Hz}, 2 \mathrm{H}, \mathrm{ArOC}_{\mathrm{H}_{\mathrm{a}}} \underline{\mathrm{H}}_{\mathrm{b}} \mathrm{CH}=\mathrm{CR}\right), 5.30$ (tq, $\left.J=3.6,1.6 \mathrm{~Hz}, 1 \mathrm{H}, \mathrm{ArOCH}_{2} \mathrm{C} \underline{\mathrm{H}}=\mathrm{CR}\right), 7.15$ (d, $J=8.4$ 
$\mathrm{Hz}, 1 \mathrm{H}, \mathrm{ArH}), 7.37-7.47$ (m, 3H, ArH), 7.76 (dd, $J=8.4,1.2 \mathrm{~Hz}, 1 \mathrm{H}, \mathrm{ArH}), 8.17$ (dd, $J=7.6,0.8$ $\mathrm{Hz}, 1 \mathrm{H}, \mathrm{ArH}) ;{ }^{13} \mathrm{C}-\mathrm{NMR}\left(\mathrm{CDCl}_{3}, 100 \mathrm{MHz}\right) \delta 26.1,37.4,69.4,121.3,121.5,122.8,125.5$, 125.7, 127.5, 127.6, 127.7, 128.7, 133.7, 134.5, 153.3; EI-MS (70 eV) m/z (rel. intensity, \%): 210 $\left(\mathrm{M}^{+}\right.$,62), 209 (30), 196 (15), 195 (100), 194 (14), 165 (15), 128 (11); HRMS (EI, m/z): Calcd. for $\mathrm{C}_{15} \mathrm{H}_{14} \mathrm{O}: 210.1045$. Found: 210.1049 .

4-Phenyl-2,5-dihydro-1-naphthoxepine (7b). (0.33 g, 60\%) was obtained as colorless crystals, $\mathrm{R}_{f}=0.85$ (ethyl acetate: $n$-hexane $=1: 7$ ); $\mathrm{mp} 95-96{ }^{\circ} \mathrm{C},{ }^{1} \mathrm{H}-\mathrm{NMR}\left(\mathrm{CDCl}_{3}, 400 \mathrm{MHz}\right) \delta 4.13(\mathrm{~s}$, $2 \mathrm{H}, \mathrm{ArOCH}_{2} \mathrm{CH}=\mathrm{CRCH}_{2}$ ), 4.93 (each $1 \mathrm{H}, \mathrm{dd}, J=3.6,3.6 \mathrm{~Hz}, 2 \mathrm{H}, \operatorname{ArOC} \underline{H}_{2} \underline{\mathrm{H}}_{\mathrm{b}} \mathrm{CH}=\mathrm{CR}$ ), 5.79 (t, $\left.J=3.6 \mathrm{~Hz}, 1 \mathrm{H}, \mathrm{ArOCH}_{2} \mathrm{CHCR}\right), 7.27$ (d, $\left.J=10.8 \mathrm{~Hz}, 1 \mathrm{H}, \mathrm{ArH}\right), 7.26-7.32(\mathrm{~m}, 1 \mathrm{H}$, $\left.\mathrm{ArOCH}_{2} \mathrm{CH}=\mathrm{CRArH}\right), 7.35-7.47$ (m, 4H, $\left.\mathrm{ArOCH}_{2} \mathrm{CH}=\mathrm{CRAr} \underline{\mathrm{H}}\right), 7.48-7.56$ (m, 3H, ArH), 7.84 $(\mathrm{d}, J=8.0 \mathrm{~Hz}, 1 \mathrm{H}, \mathrm{ArH}), 8.24(\mathrm{dd}, J=8.4,0.8 \mathrm{~Hz}, 1 \mathrm{H}, \mathrm{ArH}) ;{ }^{13} \mathrm{C}-\mathrm{NMR}\left(\mathrm{CDCl}_{3}, 100 \mathrm{MHz}\right) \delta$ $35.9,69.6,121.5,123.1,124.9,125.7,125.9,126.0,127.1,127.5,127.6,127.7,128.4,129.2$, 133.9, 138.6, 143.9, 153.2 ; EI-MS (70 eV) m/z (rel. intensity, \%): $272\left(\mathrm{M}^{+}, 55\right), 271$ (49), 258 (21), 57 (100), 253 (15), 228 (17), 195 (26); HRMS (EI, m/z): Calcd. for $\mathrm{C}_{20} \mathrm{H}_{16} \mathrm{O}: 272.1201$. Found: 272.1209.

4-(4-Methoxyphenyl)-2,5-dihydro-1-naphthoxepine (7c). (0.36 g, 60\%) was obtained as a colorless liquid, $\mathrm{R}_{f}=0.72$ (ethyl acetate: $n$-hexane $\left.=1: 7\right),{ }^{1} \mathrm{H}-\mathrm{NMR}\left(\mathrm{CDCl}_{3}, 400 \mathrm{MHz}\right) \delta 3.79(\mathrm{~s}$, $\left.3 \mathrm{H}, \mathrm{ArOCH}_{3}\right), 4.06$ (s, 2H, $\mathrm{ArOCH}_{2} \mathrm{CH}=\mathrm{CRCH}_{2}$ ), 4.88 (each $1 \mathrm{H}, \mathrm{dd}, J=3.6,3.6 \mathrm{~Hz}, 2 \mathrm{H}$, $\left.\operatorname{ArOC}_{\underline{H}_{a}} \underline{\mathrm{H}}_{\mathrm{b}} \mathrm{CH}=\mathrm{CR}\right), 5.71\left(\mathrm{t}, J=3.6 \mathrm{~Hz}, 1 \mathrm{H}, \mathrm{ArOCH}_{2} \mathrm{C} \underline{\mathrm{H}}=\mathrm{CR}\right), 6.87(\mathrm{dd}, J=6.8,2.0 \mathrm{~Hz}, 2 \mathrm{H}$, $\operatorname{ArH}), 7.25(\mathrm{~d}, J=8.4 \mathrm{~Hz}, 1 \mathrm{H}, \operatorname{ArH}), 7.33(\mathrm{dd}, J=6.8,2.0 \mathrm{~Hz}, 2 \mathrm{H}, \operatorname{ArH}), 7.41-7.51(\mathrm{~m}, 3 \mathrm{H}$, $\mathrm{ArH}), 7.79$ (d, $J=8.0 \mathrm{~Hz}, 1 \mathrm{H}, \mathrm{ArH}), 8.21$ (dd, $J=8.4,0.8 \mathrm{~Hz}, 1 \mathrm{H}, \mathrm{ArH}) ;{ }^{13} \mathrm{C}-\mathrm{NMR}\left(\mathrm{CDCl}_{3}, 100\right.$ $\mathrm{MHz}) \delta 36.0$,55.3, 69.4, 113.7, 121.5, 122.9, 123.3, 125.6, 125.8, 127.1, 127.5, 127.6, 127.7, 128.7, 133.8, 136.3, 138.4, 153.1, 158.8 ; EI-MS (70 eV) m/z (rel. intensity, \%): $302\left(\mathrm{M}^{+}, 38\right)$, 301(14), 288 (22), 287 (100), 272 (8), 244 (12); HRMS (EI, m/z): Calcd. for $\mathrm{C}_{21} \mathrm{H}_{18} \mathrm{O}_{2}$ : 302.1307. Found: 302.1300 .

\section{General procedure for the preparation of 1-allyloxynaphthalenes (9a-b)}

Under the protection of nitrogen, to a solution of 1-naphthol (1) (7.21 g, $50.0 \mathrm{mmol})$ dissolved in dry acetone $(150 \mathrm{~mL})$ was added $\mathrm{K}_{2} \mathrm{CO}_{3}(9.67 \mathrm{~g}, 70.0 \mathrm{mmol})$ followed by crotyl chloride (8a) or cinnamyl chloride $(\mathbf{8 b})(60.0 \mathrm{mmol})$, respectively. The reaction mixture was heated at reflux for 3-4 h, monitored by TLC. After cooling to room temperature, the reaction mixture was filtered to remove the solid. The filtrate which was obtained was concentrated in vacuo to remove the solvent. The resulting residue was purified by column chromatography (ethyl acetate: $n$-hexane = 1: 10) to provide pure $\mathbf{9 a - b}$, respectively.

1-(2E-Butenyloxy)naphthalene (9a). ${ }^{9}(9.14 \mathrm{~g}, 92 \%)$ was obtained as a colorless liquid, $\mathrm{R}_{f}=$ 0.75 ( ethyl acetate: $n$-hexane $=1: 7),{ }^{1} \mathrm{H}-\mathrm{NMR}\left(\mathrm{CDCl}_{3}, 400 \mathrm{MHz}\right) \delta 1.72(\mathrm{dd}, J=3.6,1.2 \mathrm{~Hz}$, $\left.3 \mathrm{H}, \mathrm{ArOCH}_{2} \mathrm{CH}=\mathrm{CHCH}_{3}\right), 4.52\left(\mathrm{dd}, J=5.6,1.2 \mathrm{~Hz}, 2 \mathrm{H}, \mathrm{ArOC}_{2} \mathrm{CH}=\mathrm{CHCH}_{3}\right), 5.73-5.89(\mathrm{~m}$, $\left.2 \mathrm{H}, \mathrm{ArOCH}_{2} \mathrm{C} \underline{\mathrm{H}}=\mathrm{CHCH}_{3}\right), 6.70(\mathrm{~d}, J=7.2 \mathrm{~Hz}, 1 \mathrm{H}, \mathrm{ArH}), 7.27-7.49$ (m, 4H, ArH), $7.73(\mathrm{dt}, J=$ 9.6, $2.8 \mathrm{~Hz}, 1 \mathrm{H}, \mathrm{ArH}), 8.28-8.32(\mathrm{~m}, 1 \mathrm{H}, \mathrm{ArH}) ;{ }^{13} \mathrm{C}-\mathrm{NMR}\left(\mathrm{CDCl}_{3}, 100 \mathrm{MHz}\right) \delta 17.8,68.7$, $104.9,120.1,122.1,125.0,125.8,126.1,126.3,126.3,127.3,129.8,134.5,154.4$; EI-MS (70 
eV) m/z (rel. intensity, \%): 198 ( $\left.\mathrm{M}^{+}, 100\right), 183$ (95), 157 (58), 145 (50), 144 (94), 116 (52), 115 (52); HRMS (EI, $m / z)$ : Calcd. for $\mathrm{C}_{14} \mathrm{H}_{14} \mathrm{O}: 198.1045$. Found: 198.1046 .

1-(3E-Phenylallyloxy)naphthalene (9b). ${ }^{10}(11.64 \mathrm{~g}, 90 \%)$ was obtained as a colorless liquid, $\mathrm{R}_{f}$ $=0.71$ (ethyl acetate: $n$-hexane $=1: 7),{ }^{1} \mathrm{H}-\mathrm{NMR}\left(\mathrm{CDCl}_{3}, 400 \mathrm{MHz}\right) \delta 4.89(\mathrm{dd}, J=5.6,1.6 \mathrm{~Hz}$, $\left.2 \mathrm{H}, \mathrm{ArOC}_{2}{ }_{2} \mathrm{CH}=\mathrm{CHC}_{6} \mathrm{H}_{5}\right), 6.54\left(\mathrm{dt}, J=16.0,5.6 \mathrm{~Hz}, 1 \mathrm{H}, \mathrm{ArOCH}_{2} \mathrm{C} \underline{\mathrm{H}}=\mathrm{CHC}_{6} \mathrm{H}_{5}\right), 6.81-6.87(\mathrm{~m}$, $\left.1 \mathrm{H}, \mathrm{ArOCH}_{2} \mathrm{CH}=\mathrm{CHC}_{6} \mathrm{H}_{5}\right), 6.89(\mathrm{~d}, J=7.2 \mathrm{~Hz}, 1 \mathrm{H}, \mathrm{ArH}), 7.25-7.52(\mathrm{~m}, 9 \mathrm{H}, \mathrm{ArH}), 7.79-7.82$ $(\mathrm{m}, 1 \mathrm{H}, \mathrm{ArH}), 8.33-8.36(\mathrm{~m}, 1 \mathrm{H}, \mathrm{ArH}) ;{ }^{13} \mathrm{C}-\mathrm{NMR}\left(\mathrm{CDCl}_{3}, 100 \mathrm{MHz}\right) \delta 68.9,105.2,120.4$, 122.1, 124.6, 125.2, 125.8, 126.4, 126.6, 127.5, 127.9, 128.6, 132.8, 134.6, 136.5, 154.4 ; EI-MS $(70 \mathrm{eV}) \mathrm{m} / \mathrm{z}$ (rel. intensity, \%): 260( $\left.\mathrm{M}^{+}, 100\right), 259(50), 245(54), 217(29), 215(24), 181(22)$, 153(17), 128(18); HRMS (EI, m/z): Calcd. for $\mathrm{C}_{19} \mathrm{H}_{16} \mathrm{O}: 260.1201$, Found: 260.1200.

\section{General procedure for the preparation of 2-allyl-1-naphthols (10a-b)}

Under the protection of dry nitrogen, 1- allyloxynaphthalenes (9a-b) $(23.0 \mathrm{mmol})$ in decalin $(30$ $\mathrm{mL}$ ) was heated to $185-190^{\circ} \mathrm{C}$ for $2.5 \mathrm{hr}$ for $\mathbf{1 0 a}$, and $0.75 \mathrm{hr}$ for $\mathbf{1 0 b}$, respectively. The reaction mixture which was obtained was distilled off the solvent by Kugehror apparatus at $4 \mathrm{mmHg}$. The resulting residue was purified by silica gel column chromatography (ethyl acetate: $n$-hexane $=1$ : 10) to give pure 10a-b, respectively.

2-(1-Methylallyl)-1-naphthol (10a). ${ }^{9} \quad(6.34 \mathrm{~g}, 80 \%)$ was obtained as a colorless liquid, $\mathrm{R}_{f}=$ 0.39 (ethyl acetate: $n$-hexane $=1: 7),{ }^{1} \mathrm{H}-\mathrm{NMR}\left(\mathrm{CDCl}_{3}, 400 \mathrm{MHz}\right) \delta 1.43(\mathrm{~d}, J=6.8 \mathrm{~Hz}, 3 \mathrm{H}$, $\left.\operatorname{ArCH}\left(\underline{\mathrm{C}}_{3}\right) \mathrm{CH}=\mathrm{CH}_{2}\right), 3.68-3.75\left(\mathrm{~m}, 1 \mathrm{H}, \operatorname{ArC} \underline{\mathrm{H}}\left(\mathrm{CH}_{3}\right) \mathrm{CH}=\mathrm{CH}_{2}\right), 5.21-5.27(\mathrm{~m}, 2 \mathrm{H}, \mathrm{ArCH}-$ $\left.\left(\mathrm{CH}_{3}\right) \mathrm{CH}=\mathrm{CH}_{2}\right), 5.77(\mathrm{~s}, 1 \mathrm{H}, \mathrm{ArOH}), 6.12(\mathrm{ddd}, J=17.2,10.4,5.6 \mathrm{~Hz}, 1 \mathrm{H}, \mathrm{ArCH}-$ $\left.\left(\mathrm{CH}_{3}\right) \mathrm{CH}=\mathrm{CH}_{2}\right), 7.22(\mathrm{~d}, J=8.4 \mathrm{~Hz}, 1 \mathrm{H}, \mathrm{ArH}), 7.38-7.44(\mathrm{~m}, 3 \mathrm{H}, \mathrm{ArH}), 7.72-7.75(\mathrm{~m}, 1 \mathrm{H}$, $\mathrm{ArH}), 8.15-8.17(\mathrm{~m}, 1 \mathrm{H}, \mathrm{ArH}) ;{ }^{13} \mathrm{C}-\mathrm{NMR}\left(\mathrm{CDCl}_{3}, 100 \mathrm{MHz}\right) \delta 18.5,38.5,114.9,120.4,121.4$, 122.8, 125.0, 125.2, 125.7, 126.1, 127.4, 133.4, 142.1, 148.9 ; EI-MS (70 eV) $\mathrm{m} / \mathrm{z}$ (rel. intensity, \%): 198 (M+100), 184 (73), 183 (100), 165 (28), 155 (47), 154 (17), 153 (30), 151 (21); HRMS (EI, $\mathrm{m} / \mathrm{z}$ ): Calcd. for $\mathrm{C}_{14} \mathrm{H}_{14} \mathrm{O}: 198.1045$. Found: 198.1047 .

2-(1-Phenylallyl)-1-naphthol (10b). ${ }^{9}(8.11 \mathrm{~g}, 78 \%)$ was obtained as a colorless liquid, $\mathrm{R}_{f}=0.67$ (ethyl acetate: $n$-hexane $=1: 7),{ }^{1} \mathrm{H}-\mathrm{NMR}\left(\mathrm{CDCl}_{3}, 400 \mathrm{MHz}\right) \delta 5.09(\mathrm{br} \mathrm{d}, J=6.8 \mathrm{~Hz}, 1 \mathrm{H}$, $\left.\operatorname{ArC} \underline{\mathrm{H}}\left(\mathrm{C}_{6} \mathrm{H}_{5}\right) \mathrm{CH}=\mathrm{CH}_{2}\right), 5.13\left(\mathrm{ddd}, J=16.8,1.6,1.6 \mathrm{~Hz}, 1 \mathrm{H}, \operatorname{ArCH}\left(\mathrm{C}_{6} \mathrm{H}_{5}\right) \mathrm{CH}=\mathrm{C}_{\mathrm{a}} \mathrm{H}_{\mathrm{b}}\right), 5.40$ (ddd, $J=10.4,1.6,1.6 \mathrm{~Hz}, 1 \mathrm{H}, \operatorname{ArCH}\left(\mathrm{C}_{6} \mathrm{H}_{5}\right) \mathrm{CH}=\mathrm{CH}_{\mathrm{a}} \underline{\mathrm{H}}_{\mathrm{b}}$ ), 5.53 (s, $\left.1 \mathrm{H}, \mathrm{ArOH}\right), 6.46$ (ddd, $J=$ 16.8, 10.0, $\left.6.4 \mathrm{~Hz}, 1 \mathrm{H}, \operatorname{ArCH}\left(\mathrm{C}_{6} \mathrm{H}_{5}\right) \mathrm{C} \underline{\mathrm{H}}=\mathrm{CH}_{2}\right), 7.21(\mathrm{~d}, J=8.4 \mathrm{~Hz}, 1 \mathrm{H}, \mathrm{ArH}), 7.26-7.39(\mathrm{~m}, 5 \mathrm{H}$, $\left.\operatorname{ArCH}\left(\mathrm{C}_{6} \underline{\mathrm{H}}_{5}\right) \mathrm{CH}=\mathrm{CH}_{2}\right), 7.43-7.51(\mathrm{~m}, 3 \mathrm{H}, \mathrm{ArH}), 7.80-7.82(\mathrm{~m}, 1 \mathrm{H}, \mathrm{ArH}), 8.16-8.19(\mathrm{~m}, 1 \mathrm{H}$, $\mathrm{ArH}) ;{ }^{13} \mathrm{C}-\mathrm{NMR}\left(\mathrm{CDCl}_{3}, 100 \mathrm{MHz}\right) \delta 50.1,117.6,120.4,121.5,121.8,125.1,125.3,126.0$, 127.1, 127.4, 127.5, 128.6, 128.9, 133.7, 139.1, 141.0, 149.0 ; EI-MS (70 eV) m/z (rel. intensity, \%): $260\left(\mathrm{M}^{+}, 100\right), 259$ (44), 245 (56), 231(23), 217 (37), 215 (25), 202 (25), 181 (20); HRMS (EI, $\mathrm{m} / \mathrm{z}$ ): Calcd. for $\mathrm{C}_{19} \mathrm{H}_{16} \mathrm{O}: 260.1201$. Found: 260.1203 .

4-(3-Phenylallyl)-1-naphthol (10c). (1.25 g, 12\%) was obtained as a yellowish brown liquid, $\mathrm{R}_{f}$ $=0.33$ (ethyl acetate: $n$-hexane $=1: 7),{ }^{1} \mathrm{H}-\mathrm{NMR}\left(\mathrm{CDCl}_{3}, 400 \mathrm{MHz}\right) \delta 3.91(\mathrm{~d}, J=5.2 \mathrm{~Hz}, 2 \mathrm{H}$, $\left.\mathrm{ArCH}_{2} \mathrm{CH}=\mathrm{CH}\right), 5.25(\mathrm{~s}, 1 \mathrm{H}, \mathrm{ArOH}), 6.43\left(\mathrm{~d}, J=16.0 \mathrm{~Hz}, 1 \mathrm{H}, \mathrm{ArCH}_{2} \mathrm{CH}=\mathrm{CH}\right), 6.49(\mathrm{dt}, J=$ 16.0, $\left.5.2 \mathrm{~Hz}, 1 \mathrm{H}, \mathrm{ArCH}_{2} \mathrm{C} \underline{\mathrm{H}}=\mathrm{CH}\right), 6.77(\mathrm{~d}, J=7.6 \mathrm{~Hz}, 1 \mathrm{H}, \operatorname{ArH}), 7.15-7.37(\mathrm{~m}, 6 \mathrm{H}$, 
$\left.\mathrm{ArCH}_{2} \mathrm{CH}=\mathrm{CHC}_{6} \underline{\mathrm{H}}_{5}, \mathrm{ArH}\right), 7.48-7.55$ (m, 2H, ArH), 8.01-8.04 (m, 1H, ArH), 8.22-8.25 (m, 1H, $\mathrm{ArH}) ;{ }^{13} \mathrm{C}-\mathrm{NMR}\left(\mathrm{CDCl}_{3}, 100 \mathrm{MHz}\right) \delta 36.0,108.2,122.2,124.1,124.8,125.0,126.1,126.2$, 126.5, 127.0, 128.5, 128.7, 129.3, 131.0, 133.0, 137.5, 150.4 ; EI-MS (70 eV) m/z (rel. intensity, \%): 260 (M+1 100), 182 (24), 181 (32), 170 (27), 169 (66), 141 (24), 128 (24), 115 (35). HRMS (EI, $\mathrm{m} / \mathrm{z}$ ): Calcd. for $\mathrm{C}_{19} \mathrm{H}_{16} \mathrm{O}: 260.1201$. Found: 260.1200 .

\section{General procedure for the preparation of 2-Allyl-1-allyloxy-naphthalenes (11a-b)}

Under the protection of nitrogen, to a solution of 2-allyl-1-naphthol (10a-b) (5.52 g, $30.0 \mathrm{mmol})$ dissolved in dry acetone $(120 \mathrm{~mL})$ was added $\mathrm{K}_{2} \mathrm{CO}_{3}(5.80 \mathrm{~g}, 42.0 \mathrm{mmol})$ followed by addng allyl bromide $(4.35 \mathrm{~g}, 36.0 \mathrm{mmol})$. The reaction mixture was heated to reflux for $3-4 \mathrm{~h}$ and monitored by TLC. After cooling to room temperature, the reaction mixture was filtered to remove the solid. The filtrate which was obtained was concentrated in vacuo to remove the solvent. The resulting residue was purified by column chromatography (ethyl acetate: $n$-hexane = 1: 10) to provide pure 11a-b, respectively.

1-Allyloxy-2-(1-methylallyl)naphthalene (11a). ${ }^{9}$ (6.18 g, 92\%) was obtained as a colorless liquid, $\mathrm{R}_{f}=0.76$, (ethyl acetate: $n$ - hexane $\left.=1: 7\right),{ }^{1} \mathrm{H}-\mathrm{NMR}\left(\mathrm{CDCl}_{3}, 400 \mathrm{MHz}\right) \delta 1.48(\mathrm{~d}, J=4.8$ $\left.\mathrm{Hz}, 3 \mathrm{H}, \operatorname{ArCH}\left(\mathrm{CH}_{3}\right) \mathrm{CH}=\mathrm{CH}_{2}\right), 4.27-4.30\left(\mathrm{~m}, 1 \mathrm{H}, \operatorname{ArCH}\left(\mathrm{CH}_{3}\right) \mathrm{CH}=\mathrm{CH}_{2}\right), 4.59(\mathrm{ddd}, J=5.6,2.8$, $\left.1.2 \mathrm{~Hz}, 2 \mathrm{H}, \operatorname{ArOC} \underline{H}_{2} \mathrm{CH}=\mathrm{CH}_{2}\right), 5.13-5.19\left(\mathrm{~m}, 2 \mathrm{H}, \operatorname{ArCH}\left(\mathrm{CH}_{3}\right) \mathrm{CH}=\underline{\mathrm{CH}}_{2}\right), 5.41$ (ddt, $J=10.4$, 1.2, $\left.1.2 \mathrm{~Hz}, 1 \mathrm{H}, \mathrm{ArOCH}_{2} \mathrm{CH}=\mathrm{CH}_{\mathrm{a}} \mathrm{H}_{\mathrm{b}}\right) 5.61$ (ddt, $J=17.2,1.2,1.2 \mathrm{~Hz}, 1 \mathrm{H}, \mathrm{ArOCH}_{2} \mathrm{CH}=\mathrm{CH}_{\mathrm{a}} \underline{\mathrm{H}}_{\mathrm{b}}$ ), 6.13-6.21 (m, 1H, $\left.\operatorname{ArOCH}{ }_{2} \underline{\mathrm{H}}=\mathrm{CH}_{2}\right), 6.25-6.34\left(\mathrm{~m}, 1 \mathrm{H}, \operatorname{ArCH}\left(\mathrm{CH}_{3}\right) \mathrm{C} \underline{H}=\mathrm{CH}_{2}\right), 7.41(\mathrm{dd}, J=$ 8.8, $2.0 \mathrm{~Hz}, 1 \mathrm{H}, \mathrm{ArH}), 7.48-7.58$ (m, 2H, ArH), 7.68 (d, $J=8.8 \mathrm{~Hz}, 1 \mathrm{H}, \mathrm{ArH}), 7.87$ (dt, $J=8.4$, $0.8 \mathrm{~Hz}, 1 \mathrm{H}, \mathrm{ArH}), 8.18$ (dt, $J=8.4,0.8 \mathrm{~Hz}, 1 \mathrm{H}, \mathrm{ArH}) ;{ }^{13} \mathrm{C}-\mathrm{NMR}\left(\mathrm{CDCl}_{3}, 100 \mathrm{MHz}\right) \delta 20.5,35.3$, 75.6, 113.4, 117.3, 122.2, 124.3, 125.6, 125.6, 125.9, 127.9, 128.2, 133.6, 133.7, 133.9, 143.0, 151.3; EI-MS (70 eV) m/z (rel. intensity, \%): $238\left(\mathrm{M}^{+}, 100\right), 223$ (22), 196 (16), 194 (12), 182 (18), 181 (31), 179 (15), 165 (11); HRMS (EI, m/z): Calcd. for $\mathrm{C}_{17} \mathrm{H}_{18} \mathrm{O}$ : 238.1358, Found: 238.1356.

1-Allyloxy-2-(1-phenylallyl)naphthalene (11b). (8.19 g, 91\%), was obtained as a colorless liquid, $\mathrm{R}_{f}=0.73$ (ethyl acetate: $n$-hexane $\left.=1: 7\right),{ }^{1} \mathrm{H}-\mathrm{NMR}\left(\mathrm{CDCl}_{3}, 400 \mathrm{MHz}\right) \delta 4.44-4.59(\mathrm{~m}$, $\left.2 \mathrm{H}, \quad \operatorname{ArOC} \underline{H}_{2} \mathrm{CH}=\mathrm{CH}_{2}\right), \quad 5.08-5.16\left(\mathrm{~m}, 1 \mathrm{H}, \quad \operatorname{ArCH}\left(\mathrm{C}_{6} \mathrm{H}_{5}\right) \mathrm{CH}=\mathrm{CH}_{2}\right), \quad 5.34-5.41 \quad(\mathrm{~m}, 2 \mathrm{H}$, $\left.\operatorname{ArCH}\left(\mathrm{C}_{6} \mathrm{H}_{5}\right) \mathrm{CH}=\underline{\mathrm{CH}}_{2}\right), 5.57\left(\mathrm{ddt}, J=10.4,1.6,1.6 \mathrm{~Hz}, 1 \mathrm{H}, \mathrm{ArOCH}_{2} \mathrm{CH}=\mathrm{C}_{\mathrm{a}} \mathrm{H}_{\mathrm{b}}\right), 5.60$ (ddt, $J=$ 16.0, 1.6, 1.6 Hz, 1H, $\left.\mathrm{ArOCH}_{2} \mathrm{CH}=\mathrm{CH}_{\mathrm{a}} \underline{\mathrm{H}}_{\mathrm{b}}\right), 6.21-6.32\left(\mathrm{~m}, 1 \mathrm{H}, \mathrm{ArOCH}_{2} \mathrm{CH}=\mathrm{CH}_{2}\right), 6.41-6.50(\mathrm{~m}$, $\left.1 \mathrm{H}, \operatorname{ArCH}\left(\mathrm{C}_{6} \mathrm{H}_{5}\right) \mathrm{C} \underline{\mathrm{H}}=\mathrm{CH}_{2}\right), 7.25-7.29(\mathrm{~m}, 1 \mathrm{H}, \mathrm{ArH}), 7.35-7.38\left(\mathrm{~m}, 5 \mathrm{H}, \operatorname{ArCH}\left(\mathrm{C}_{6} \underline{\mathrm{H}}_{5}\right) \mathrm{CH}=\mathrm{CH}_{2}\right)$, 7.50-7.59 (m, 2H, ArH), 7.66 (d, J=8.8 Hz, 1H, ArH), 7.88 (d, J=8.0 Hz, 1H, ArH), 8.20 (d, $J$ $=8.0 \mathrm{~Hz}, 1 \mathrm{H}, \mathrm{ArH}),{ }^{13} \mathrm{C}-\mathrm{NMR}\left(\mathrm{CDCl}_{3}, 100 \mathrm{MHz}\right) \delta 47.1,75.4,116.8,117.2,122.3,124.2$, $125.8,125.9,126.2,127.2,127.9,128.3,128.5,131.5,133.8,133.8,140.5,143.2,152.1$; EI-MS (70 eV) m/z (rel. intensity, \%): 300 (M+1 100), 298 (25), 259 (63), 209 (59), 207 (20), 182 (20), 181 (51), 165 (24); HRMS (ESI, m/z): Calcd. for $\mathrm{C}_{22} \mathrm{H}_{20} \mathrm{ONa}$ : 323.1412, Found: 323.1415. 
General procedure for the preparation of 5-substituted 2,5-dihydro-1-naphthoxepines (12ab)

Under the protection of dry nitrogen, 2-allyl-1-allyloxy-naphthalenes (11a-c) (2.0 mmol) dissolved in dichloromethane $(200 \mathrm{~mL})$ was stirred and added Grubbs' catalyst (II) (0.085 g, $5 \% \mathrm{~mol}$. The reaction mixture was continually stirred at room temperature for $24 \mathrm{~h}$ until the consumption of starting material which was monitored by TLC. After filtration, the filtrate was concentrated in vacuo to remove the solvent. The resulting residue was purified by column chromatography (ethyl acetate: $n$-hexane $=1: 50$ ) to give pure 12a-c, respectively.

5-Methyl-2,5-dihydro-1-naphthoxepine (12a). (0.38 g, 60\%) was obtained as a colorless liquid, $\mathrm{R}_{f}=0.74$ (ethyl acetate: $n$-hexane $\left.=1: 7\right),{ }^{1} \mathrm{H}-\mathrm{NMR}\left(\mathrm{CDCl}_{3}, 400 \mathrm{MHz}\right) \delta 1.52(\mathrm{~d}, J=7.2$ $\left.\mathrm{Hz}, 3 \mathrm{H}, \operatorname{ArCH}\left(\mathrm{CH}_{3}\right) \mathrm{CH}=\mathrm{CH}\right), 3.68-3.72\left(\mathrm{~m}, 1 \mathrm{H}, \operatorname{ArC} \underline{\mathrm{H}}\left(\mathrm{CH}_{3}\right) \mathrm{CH}=\mathrm{CH}\right), 4.54-4.76(\mathrm{~m}, 2 \mathrm{H}$, $\left.\operatorname{ArOC}_{2}{ }_{2} \mathrm{CH}=\mathrm{CH}\right), \quad 5.48-5.52 \quad\left(\mathrm{~m}, \quad 1 \mathrm{H}, \quad \operatorname{ArOCH}_{2} \mathrm{CH}=\mathrm{CH}\right), \quad 5.84-5.90 \quad(\mathrm{~m}, \quad 1 \mathrm{H}$, $\left.\mathrm{OCH}=\mathrm{CHCH}\left(\mathrm{CH}_{3}\right) \mathrm{Ar}\right), 7.24(\mathrm{~d}, J=8.4 \mathrm{~Hz}, 1 \mathrm{H}, \mathrm{ArH}), 7.41-7.50(\mathrm{~m}, 2 \mathrm{H}, \mathrm{ArH}), 7.53(\mathrm{~d} . J=8.0$ $\mathrm{Hz}, 1 \mathrm{H}, \mathrm{ArH}), 7.80(\mathrm{~d}, J=8.0 \mathrm{~Hz}, 1 \mathrm{H}, \mathrm{ArH}), 8.20(\mathrm{dd}, J=8.4,0.8 \mathrm{~Hz}, 1 \mathrm{H}, \mathrm{ArH}) ;{ }^{13} \mathrm{C}-\mathrm{NMR}$ $\left(\mathrm{CDCl}_{3}, 100 \mathrm{MHz}\right) \delta 21.6,38.1,69.7,121.5,123.6,125.6,125.9,126.2,126.3,127.6,128.1$, 132.2, 133.7, 135.6, 152.7 ; EI-MS (70 eV) m/z (rel. intensity, \%): 210( $\left.\mathrm{M}^{+}, 100\right), 197(13)$, 196(46), 195.7(59), 195(33), 165(17); HRMS (EI, m/z): Calcd. for $\mathrm{C}_{15} \mathrm{H}_{14} \mathrm{O}$ : 210.1045. Found: 210.1048 .

5-Phenyl-2,5-dihydro-1-naphthoxepine (12b). (0.45 g, 55\%) was obtained as a colorless liquid, $\mathrm{R}_{f}=0.68$ (ethyl acetate: $n$-hexane $\left.=1: 7\right),{ }^{1} \mathrm{H}-\mathrm{NMR}\left(\mathrm{CDCl}_{3}, 400 \mathrm{MHz}\right) \delta 4.62-4.69(\mathrm{~m}, 1 \mathrm{H}$, $\left.\mathrm{CH}=\mathrm{CHC} \underline{\mathrm{H}}\left(\mathrm{C}_{6} \mathrm{H}_{5}\right) \mathrm{Ar}\right), \quad 4.86-4.92 \quad\left(\mathrm{~m}, \quad 2 \mathrm{H}, \quad \operatorname{ArOC}_{2}{ }_{2} \mathrm{CH}=\mathrm{CHCH}\right), \quad 5.77-5.82 \quad(\mathrm{~m}, \quad 1 \mathrm{H}$, $\left.\mathrm{ArOCH}_{2} \mathrm{CH}=\mathrm{CHCH}\right), 6.10-6.16\left(\mathrm{~m}, 1 \mathrm{H}, \mathrm{CH}=\mathrm{CHCH}\left(\mathrm{C}_{6} \mathrm{H}_{5}\right) \mathrm{Ar}\right), 7.18-7.32(\mathrm{~m}, 5 \mathrm{H}, \mathrm{CH}=\mathrm{CHCH}-$ $\left.\left(\mathrm{C}_{6} \underline{\mathrm{H}}_{5}\right) \mathrm{Ar}\right), 7.43-7.52(\mathrm{~m}, 3 \mathrm{H}, \mathrm{ArH}), 7.54(\mathrm{~d}, J=8.8 \mathrm{~Hz}, 1 \mathrm{H}, \mathrm{ArH}), 7.80(\mathrm{dd}, J=8.8,1.2 \mathrm{~Hz}, 1 \mathrm{H}$, $\mathrm{ArH}), 8.22(\mathrm{dd}, J=8.8,0.8 \mathrm{~Hz}, 1 \mathrm{H}, \mathrm{ArH}) ;{ }^{13} \mathrm{C}-\mathrm{NMR}\left(\mathrm{CDCl}_{3}, 100 \mathrm{MHz}\right) \delta 50.1,69.7,121.8$, $123.7,125.9,126.0,126.4,127.5,127.6,127.9,128.1,128.2,128.5,129.4,133.5,133.9,143.0$, 152.7 ; EI-MS (70 eV) m/z (rel. intensity, \%): $272\left(\mathrm{M}^{+}, 100\right), 271$ (61), 257 (96), 228 (29), 215 (23), 195 (58), 181 (48), 165 (33); HRMS (ESI, m/z): Calcd. for $\mathrm{C}_{20} \mathrm{H}_{16} \mathrm{ONa}$ : 295.1099, Found: 295.1101.

\section{Acknowledgements}

Financial support (Grant no., NSC-96-2113-M-037-006) from NSC, Taiwan is grateful.

\section{References}

1. (a) Huang, K. S.; Wang, E. C. Tetrahedron Lett. 2001, 42, 6155. (b) Huang, K. S.; Wang, E. C.; Chen, H. M. J. Chin. Chem. Soc. 2004, 51, 585. (c) Huang, K. S.; Wang, E. C.; Chen, H. M. J. Chin, Chem. Soc. 2004, 51, 807. (d) Huang, K. S.; Wang, E. C. J. Chin. Chem. Soc. 
2004, 51, 383. (e) Chen, P. Y.; Chen, H. M.; Chen, L. Y.; Tzeng, J. C.; Chi, P. C.; Li, S. R.; Wang, E. C. Tetrahedron 2007, 63, 2824. (f) Wang, E. C.; Hsu, M. K.; Lin, Y. L.; Huang, K. S. Hetrocycles 2002, 57,1997. (g) Wang, E. C.; Wang, C. C.; Hsu, M. K.; Huang, K. S. Hetreocycles 2002, 57, 2021. (h) Tsai, T. W.; Wang, E. C.; Huang, K. S.; Li, S. R.; Wang, Y. F.; Lin, Y. L.; Chen, Y. H. Heterocycles 2004, 63, 1771. (i) Tsai, T. W.; Wang, E. C.; Li, S. R.; Chen, Y. H.; Lin, Y. L.; Wang, Y. F.; Huang, K. S. J. Chin. Chem. Soc. 2004, 51, 1307. (j) Huang, K. S.; Li, S. R.; Wang, Y. F.; Lin, Y. L.; Chen, Y. H.; Tsai, T. W.; Yang, C. H.; Wang, E. C. J. Chin. Chem. Soc. 2005, 52, 159. (k) Wang, E. C.; Lin, Y. L.; Chen, H. M.; Li, S. R.; Tsai, J. C.; Kabuto, C.; Takeuchi, Y. Heterocycles 2006, 68, 125. (1) Li, S. R.; Chen, L. Y.; Tsai, J. C.; Tzeng, J. Y.; Tsai, I. L.; Wang, E. C. Tetrahedron Lett. 2007, 48, 2139. (m) Li, S. R.; Chen, H. M.; Chen, L. Y.; Tsai, J. C.; Chen, P. Y.; Hsu, S. C. N.; Wang, E. C. ARKIVOC 2008, 172.

2. Kotha, S.; Mandal, K.;Tiwari, A.; Mobin, S. M. Chem. Euro. J. 2006, 12, 8024.

3. (a) Buu-Hoi, N. P.; Saint-Ruf, G.; Perche, J. C. J. Chem. Soc. 1970, 1327. (b) Jonas, J.; Forrest, T. P. J. Org. Chem. 1970, 35, 836. (c) Cagniant, P.; Charaux, C. Bull. Soc. Chim. Fr. 1966, 3249. (d) Cagniant, D.; Charaux, C.; Cagniant, P. Bull. Soc. Chim. Fr. 1966, 3644. (e) Chattopadhyay, S. K.; Ghosh, D.; Neogi, K. Synth. Commun. 2007, 37, 1535.

4. Dowell, A. M., Jr.; McCullough, H. S.; Calaway, P.K. J. Am. Chem. Soc. 1948, 70, 226.

5. Malmusi, L.; Franchini, S.; Mucci, A.; Schenetti, L.; Gulini, U.; Marucci, G.; Brasili, L. Bioorg. Med. Chem. 1998, 6, 825.

6. Donnelly, J. A.; Macken, P. J.; O'Brien, S. Israel J. Chem. 1981, 21, 185.

7. Kongkathip, N.; Kongkathip, B.; Siripong, P.; Sangma, C.; Luangkamin, S.; Niyomdecha, M.; Pattanapa, S.; Piyaviriyagul, S.; Kongsaeree, P. Bioorg. Med. Chem. 2003, 11, 3179.

8. Barluenga, J.; Fananas, F. J.; Sanz, R.; Marcos, C.; Ignacio, J. M. Chem. Commun. 2005, 933.

9. Ollevier, T.; Mwene-Mbeja, T. M. Tetrahedron Lett. 2006, 47, 4051, and literatures cited therein.

10. Xu, C.; Lu, S.; Huang, X. Synth. Commun. 1993, 23, 2527. 